\author{
D. NETTELS \\ R. MÜLLER-SIEBERT \\ A. WEIS
}

\title{
Relaxation mechanisms of multi-quantum coherences in the Zeeman structure of atomic Cs trapped in solid He
}

\author{
Département de Physique, Université de Fribourg, Chemin du Musée 3, 1700 Fribourg, Switzerland
}

\begin{abstract}
Received: 23 July 2003
Published online: 4 November 2003 • (C) Springer-Verlag 2003

ABSTRACT This paper extends our previous work on neardegenerate magnetic resonance transitions in alkali ground states involving the simultaneous absorption of multiple radiofrequency quanta. New experimental results with an improved spectral resolution were obtained with cesium atoms trapped in the cubic phase of a helium crystal. The main objective of the paper is a theoretical study of the influence of stochastic perturbations of given multipole orders on the various multi-photon coherences. Algebraic and numerical results for perturbations of both dipolar and quadrupolar symmetry are presented. The present experimental resolution does not yet allow us to distinguish between these two most likely relaxation mechanisms. Nonetheless, the experimental spectra are very well described when allowing in the calculations for a magnetic field inhomogeneity of $2 \times 10^{-5}$.
\end{abstract}

PACS 76.70.Hb; 32.80.Wr; 32.30.Dx; 32.60.+i

1

Introduction

In a recent paper the observation of magnetic resonance transitions involving the simultaneous absorption of $N$ radio frequency (rf) photons between Zeeman split hyperfine sublevels $|F, M\rangle$, and $|F, M+N\rangle$, in the $6 S_{1 / 2}$ ground state of Cs implanted in the body-centered cubic (b.c.c.) phase of solid $\mathrm{He}^{4}[1]$ was reported. Such transitions are of multiple interest regarding applications and fundamental investigations. Under the simplest model assumption that all multi-quantum coherences have the same relaxation rate, one expects the $N$-photon transitions to have a resonance linewidth which is $N$-times smaller than the width of usual one-photon transition. Therefore multi-photon transitions have the potential to increase the spectroscopic sensitivity in magnetic resonance experiments. It was shown [1] that some specific transitions allow a suppression of a serious systematic effect connected with quadratic Stark shifts in experiments searching for permanent electric dipole moments. Finally it was suggested [1] that multiphoton spectra might be a useful tool for the better understanding of the mechanisms, which govern the relaxation

Fax: +41-26/300-9631, E-mail: daniel.nettels@unifr.ch of the ground state spin polarization of the cesium atoms due to their interactions with lattice vibrations of the surrounding He matrix.

The theoretical investigation of the influence of different relaxation processes on the multi-photon spectra and the comparison with improved experimental data are the main topic of the present paper. In our previous work [1] only relaxation based on optical pumping was taken into account, similar to the calculations of Pazgalev and Alexandrov [2]. This work has significantly extended the treatment of the relaxation processes using generalized ground state master equations describing the evolution of the atomic ground state populations and coherences in the density matrix formalism under the influence of static and oscillating magnetic fields, optical pumping and various relaxation processes.

The optical absorption and emission lines of Cesium atoms embedded in the body-centered cubic (b.c.c.) phase of solid $\mathrm{He}^{4}$ are strongly broadened and shifted by the helium matrix [3]. As a consequence of the very high degree of spherical symmetry of the local trapping sites of the impurity atoms (atomic bubbles) the sample can nonetheless be efficiently polarized by optical pumping [4]. The nonmagnetic nature of the host matrix atoms and their isotropic distribution around the defect atoms ensure that the alkali spins are only very weakly perturbed, which is reflected by longitudinal spin relaxation times $T_{1}=1 / \gamma_{1}$ of $\left\langle J_{z}\right\rangle$ on the order of 1 second [5] and by very narrow magnetic resonance lines [6]. For a static distribution of the helium atoms around the cesium atoms one would expect quasiinfinite relaxation times. The very long, but nonetheless finite longitudinal and transverse spin coherence times are most probably due to fluctuations of the bubble interface boundary. So far no detailed investigations of the mechanisms by which such fluctuations couple to the Cs spins were performed. The present paper calculates the effect of dipolar and quadrupolar shape oscillations on the relaxation of multi-photon coherences, and shows explicitly that the spectra have the potential to discriminate between the two processes.

The previously measured magnetic resonance lines were strongly broadened due to magnetic field inhomogeneities and current instabilities. This broadening severely hinders the study of the intrinsic relaxation mechanisms. In the meantime 
the experimental apparatus has been improved by suppressing some of these technical noise sources. New experimental data is presented showing an increased spectroscopic resolution. As before the measurements were done using the technique of optically detected magnetic resonance (ODMR), in which resonant optical interactions are used both to build up spin polarization in the sample, and to detect radio-frequency induced transitions between the magnetic sublevels via their effect on the optical properties of the alkali sample. Unfortunately the observed widths of the experimental spectra are still inhomogeneously broadened and do not yet allow a conclusion to be drawn regarding the actual relaxation mechanism. Nonetheless the spectra can be very well reproduced by allowing for a small field inhomogeneity in the calculations.

\section{Simple theory of multi-photon transitions \\ 2.1 Multi-photon transition between substrates of a hyperfine level $F$ in a magnetic field}

A detailed introduction to the structure of Zeemansplit multi-photon transitions was presented in previous work [1]. The main features are recalled here. In a weak magnetic field $B_{0}$ (linear Zeeman effect) all $\Delta M=1$ single photon transitions between adjacent levels $|F, M\rangle$ and $|F, M+1\rangle$ of an isolated hyperfine multiplet $F$ have the same transition frequency $\omega_{L}=\gamma B_{0}=g_{F} \mu_{B} B_{0} / \hbar$. In stronger magnetic fields the combined hyperfine and Zeeman interactions lead to level shifts which are nonlinear in $B_{0}$ and $M$ (Breit-Rabi diagram). The lowest order correction terms to the linear Zeeman effect are quadratic in $M$ and can be parameterized as

$\omega_{M}=\omega_{\mathrm{hfs}}+\gamma M B_{0}+\left(\alpha+\beta M^{2}\right) B_{0}^{2}$,

where $\omega_{\mathrm{hfs}}$ is the hyperfine frequency shift of the given hyperfine level $F$, and $\alpha$ and $\beta$ are constants depending on the specific state. As a consequence of the non-equidistant level spacing processes in which $N$ identical rf-photons are simultaneously absorbed can be spectrally resolved. The resonance frequency of an $N$-photon transition starting from level $M$ is given by

$\frac{\omega_{M+N}-\omega_{M}}{N}=\gamma B_{0}+\beta B_{0}^{2}(2 M+N)$.

There are $2 F$ distinct transitions involving the absorption of a single photon transitions, $2 F-1$ two photon transitions ..., up to a single transition involving the absorption of $N=2 F$ photons. The resonance energies of all possible multi-photon transitions are represented in Fig. 1 for the hyperfine level $F=4$. The higher order multiphoton lines appear successively in the magnetic resonance spectra as the rf intensity is increased.

\subsection{Lineshapes of multi-quantum transitions}

The simplest system which allows the observation of multi-quantum transitions is the $F=1$ three level system shown in Fig. 2.

Multi-photon transitions in that system were treated by Salwen [7], and also by Hermann and Swain [8]. In both references relaxation processes were not taken into account and $\mathrm{rf}$

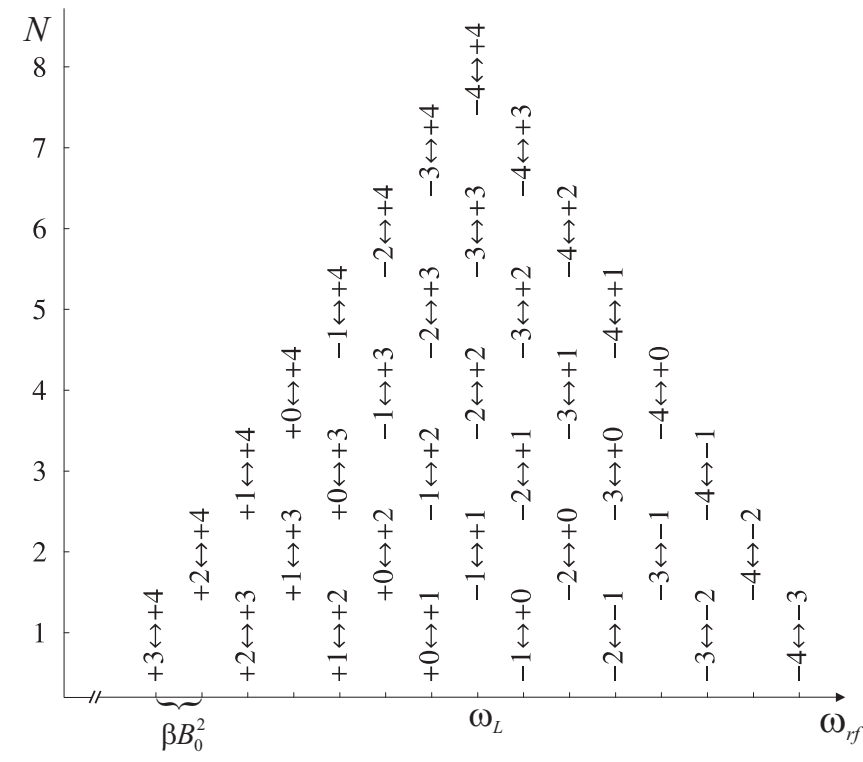

FIGURE 1 Resonance frequencies of the multi-photon transitions involving $N=1 \ldots 8$-photons coupling states $M$ and $M+N$ in a spin $F=4$ system. The line positions are given by (1) with $\beta<0$ as for Cs

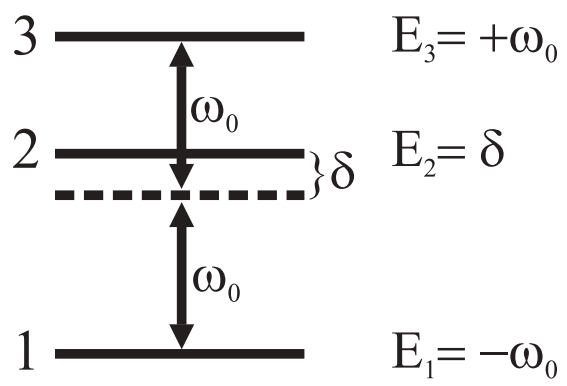

FIGURE 2 Three-level system: level 2 is detuned by $\delta$ from the average energy spacing $\omega_{0}=\frac{1}{2}\left(E_{3}-E_{1}\right)$ of levels 1 and 2

power broadening was the only mechanism which determined the resonance line shapes. Under the assumption that the resonance lines are spectrally well resolved the lineshapes of the multi-quantum transitions can then be approximated by simple Lorentzians. The calculations of these authors have been extended by explicitly adding relaxation terms for the different multi-photon coherences.

The interaction with an rf-field with frequency $\omega_{\mathrm{rf}}$ is described in the rotating wave approximation by the time independent Hamiltonian

$H=\hbar\left(\begin{array}{ccc}\Delta & \Omega_{12} & 0 \\ \Omega_{12}^{*} & \delta & \Omega_{23} \\ 0 & \Omega_{23}^{*} & -\Delta\end{array}\right)$

where $\Delta=\omega_{\mathrm{rf}}-\omega_{0} . \delta$ is the displacement of the intermediate state. For simplicity it is assumed that the Rabi frequencies $\Omega_{12}=\Omega_{23} \equiv \Omega=\Omega^{*}$. Making the additional assumptions that the populations of all states relax with a common rate $\gamma_{1}$ against a steady state population and that the $\Delta M=1$ coherences $\varrho_{12}$ and $\varrho_{23}$ relax with $\gamma_{2}$, and that the $\Delta M=2$ coherence $\varrho_{13}$ relaxes with $\gamma_{3}$, we obtain the following approximative expressions for the lineshapes of the transitions 
$1-2$ and $1-3$ :

$$
\begin{aligned}
& P_{12}\left(\Delta_{1}\right)=\frac{2 \frac{\gamma_{2}}{\gamma_{1}} \Omega^{2}}{\Delta_{1}^{2}+\gamma_{2}^{2}+4 \frac{\gamma_{2}}{\gamma_{1}} \Omega^{2}} \\
& P_{13}\left(\Delta_{2}\right)=\frac{2 \frac{\gamma_{3}}{\gamma_{1}} \frac{\Omega^{2}}{2} \frac{\Omega^{2}}{\delta}}{\Delta_{2}^{2}+{\frac{\gamma_{3}}{2}}^{2}+4 \frac{\gamma_{3}}{\gamma_{1}} \frac{\Omega^{2}}{2} \frac{\Omega^{2}}{\delta}},
\end{aligned}
$$

where $\Delta_{1}$ and $\Delta_{2}$ are the detunings from the one-photon and two-photon transition frequencies respectively.

In a next step the calculation extended to spin systems is with arbitrary values of $F$ in which $N$-photon transitions occur, connecting states $|F, M\rangle$ and $|F, M+N\rangle$. For the sake of simplicity it is assumed that the intermediate states $\mid F, M+$ $k\rangle$ with $(k=2, \ldots, N-1)$ are all displaced by the same amount $\delta$ from the value $\omega_{M}+k \omega_{0}^{(N)}$, where $\omega_{0}^{(N)}=\left(\omega_{M+N}-\omega_{M}\right) / N$. Again we set all $\Omega_{i j}=\Omega$ to get

$$
P_{M, M+N}=\frac{2 \frac{\gamma_{N+1}}{\gamma_{1}} \frac{\Omega^{2}}{N} \frac{\Omega}{\delta}^{2(N-1)}}{\Delta_{N}^{2}+{\frac{\gamma_{N+1}}{N}}^{2}+4 \frac{\gamma_{N+1}}{\gamma_{1}} \frac{\Omega^{2}}{N} \frac{\Omega}{\delta}^{2(N+1)}},
$$

where $\Delta_{N}$ is the detuning from the $N$-photon resonance and $\gamma_{N+1}$ is the relaxation rate of the coherence between the states $|M\rangle$ and $|M+N\rangle$.

Although in the experiments the condition (spectral isolation of the $N$-photon resonance lines) for the validity of (2) is not completely fulfilled, it is nonetheless instructive to use the simple Lorentzian lineshapes of (2) for a first qualitative comparison of the multi-photon lines with the single-photon lines. Particular interest lies in the possible achievable gain in spectroscopic sensitivity. This sensitivity is determined by the accuracy with which the center of a given resonance line can be determined experimentally at a given experimental noise level, and is proportional to the steepness of the resonance, i.e., the maximal slope $|d P / d \Delta|$ of the resonance lineshape $P(\Delta)$. The steepness is proportional to the ratio $R_{N}=P(\Delta=0) / \Delta \omega_{\text {FWHM }}$ of the resonant signal amplitude and its width. For the Lorentzians of (2) the resonant transition probability $P(0)$ of an $N$-photon transition reaches a maximum value of

$R_{N}^{\max }=\frac{1}{6 \sqrt{3}} \frac{N}{\gamma_{N+1}}$

at the optimal rf amplitude given in terms of the corresponding Rabi frequency by

$\Omega=\Omega_{\mathrm{opt}} \equiv \delta\left(\frac{\gamma_{1} \gamma_{N+1}}{2 \delta^{2}}\right)^{\frac{1}{2 N}}$,

The corresponding transition probabilities $P_{M, M+N}\left(\Delta_{N}=\right.$ $0, \Omega=\Omega_{\text {opt }}$ ) are found to have the same constant value of $1 / 3$ independent of $N$ and the widths of the Lorentzians are found to vary with $N$ as

$\Delta \omega_{\mathrm{FWHM}}=2 \sqrt{3} \frac{\gamma_{N+1}}{N}$, so that the widths alone determine the $N$-dependence of the steepness. The validity of the used approximations requires that the relaxation rates $\gamma_{i}$ are much smaller than $\delta$. Hence $\Omega$ has to be increased according to (4) in order to observe the higher order multi-photon resonances. The relative change of the sensitivity of an $N$-photon resonance compared to a single photon resonance is thus given according to (3) by

$G_{N} \equiv \frac{R_{N}^{\max }}{R_{N=1}^{\max }}=\frac{\gamma_{2}}{\gamma_{N+1}} N$.

For the simplest case, treated in the previous paper, in which all multi-photon relaxation rates $\gamma_{2}, \gamma_{3}, \ldots, \gamma_{N+1}$ are equal, one thus obtains an increased sensitivity, which is directly proportional to the number of involved photons. However, in the more realistic case, in which the rates $\gamma_{N}$ differ this simple scaling law no longer holds. It is one of the goals of this paper to determine how the relaxation rates $\gamma_{N}$ of multiquantum coherences and hence the anticipated improvement of the spectroscopic sensitivity $G_{N}$ depend on the nature and symmetry of different relaxation mechanisms.

\section{Numerical Calculation of the ODMR signal 3.1 Optically detected magnetic resonance}

In the experiments the magnetic resonances addressed in the previous paragraph were observed using a double resonance technique. A circularly polarized laser beam resonant with the Cs $D_{1}$-transition was used to prepare the necessary population differences in the magnetic sublevels by optical pumping. As the scattering rate of the laser radiation depends on the degree of spin polarization of the medium, any change of the latter due to a magnetic resonance transition can be detected by monitoring the fluorescence light intensity. This technique is known as optically detected magnetic resonance (ODMR).

The multi-photon ODMR spectra of Cs has been calulated by numerically solving the steady-state Liouville equation for the density matrix $\varrho$ describing the 16 sublevels of the $6 S_{1 / 2}$ ground state hyperfine structure $(F=3,4)$. The rate of change $\dot{\varrho}$ of the density matrix is governed by the interaction with the (static and oscillating) magnetic fields, the optical pumping interaction with the resonant circularly polarized laser beam and the sublevel population and coherence relaxation processes:

$\dot{\varrho}=(\dot{\varrho})_{\mathrm{mag}}+(\dot{\varrho})_{\mathrm{op}}+(\dot{\varrho})_{\mathrm{rel}}$.

Steady-state solutions were obtained by setting $\dot{\varrho}=0$. The following discusses the three terms of (6) in detail.

\subsection{Magnetic interaction}

The Liouville equation is solved in the $\left|\left(F_{ \pm}\right), M\right\rangle$ basis in which the Hamiltonian

$H_{0}=A \boldsymbol{I} \cdot \boldsymbol{J}+g_{J} \mu_{B} J_{z} B_{0}-g_{I} \mu_{B} I_{z} B_{0}$,

describing the hyperfine and static Zeeman interactions is diagonal. The brackets around $F_{ \pm}$in $\left|\left(F_{ \pm}\right), M\right\rangle$ indicate that $F$ is not a good quantum number in the presence of the magnetic 
field; $F_{ \pm}$are merely used as convenient state labels. Since the two hyperfine levels are well separated in energy, it is justified to neglect all matrix elements of the radio frequency interaction operator which couple states of different $F$-values. The corresponding coherences in the density matrix $\varrho$ will also be ignored, so that the density matrix of the ground state has the form

$\varrho=\left(\begin{array}{cc}\varrho_{F_{-}} & 0 \\ 0 & \varrho_{F_{+}}\end{array}\right)$,

where $\varrho_{F_{ \pm}}$are sub-matrices restricted to the eigenspaces of $F_{ \pm}$spanned by the states $\left|\left(F_{ \pm}\right), M\right\rangle$. The interaction operator of the atoms with the rf-field $B_{1}(t)=B_{1} \cos \omega t$ oscillating along the $x$-direction is

$$
\begin{aligned}
V(t) & =\left(g_{J} \mu_{B} J_{x} B_{1}-g_{I} \mu_{B} I_{x} B_{1}\right) \cos \omega_{\mathrm{rf}} t \\
& =2 V_{0} \cos \omega_{\mathrm{rf}} t .
\end{aligned}
$$

In the rotating field approximation the Liouville equations for $\varrho_{F_{-}}$and $\varrho_{F_{+}}$are

$i \hbar\left(\dot{\varrho}_{F_{ \pm}}\right)_{\mathrm{mag}}=\left[H_{ \pm}, \varrho_{F_{ \pm}}\right]$

with the time-independent Hamiltonian

$H_{ \pm}=H_{0} \mp \hbar \omega_{\mathrm{rf}} F_{z}+V_{0}$,

where all operators are restricted to the corresponding $\left|\left(F_{ \pm}\right), M\right\rangle$ spaces. The different signs in front of the second term reflect the facts that $\omega_{\mathrm{rf}}$ has been chosen to be positive, and that the $g$-factors of the two hyperfine levels have opposite signs.

\subsection{Optical pumping and detection signal}

A detailed discussion of optical pumping processes of Cs atoms in solid ${ }^{4} \mathrm{He}$ can be found in [4]. In that paper optical pumping was calculated using rate equations for the ground state populations. This approach is generalized by the simultaneous treatment of optical pumping and ground state coherence evolution under the action of the magnetic fields. The destruction of these coherences by the absorptive interaction with the optical radiation then contributes to the broadening of the magnetic resonance lines. In the experiment the laser beam traverses several cryostat windows, some of which show stress-induced birefringence. As a consequence the beam interacting with the sample is not $100 \%$ circularly polarized. This is taken into account by allowing the light field $\boldsymbol{E}$ to be elliptically polarized

$$
\begin{aligned}
\boldsymbol{E} & =\frac{E_{0}}{\sqrt{2}}\left(\begin{array}{c}
\cos \omega t \\
\sin \omega t+\varphi
\end{array}\right)=\frac{E_{0}}{\sqrt{2}}\left(\begin{array}{c}
1 \\
i e^{-i \varphi}
\end{array}\right) \frac{\mathrm{e}^{-i \omega t}}{2}+\text { c.c. } \\
& =\frac{1}{2} \& \mathrm{e}^{-i \omega t}+\text { c.c. },
\end{aligned}
$$

where c.c. denotes the complex conjugate, $\omega$ is the light frequency and $E_{0}$ is the amplitude of the electric field. The two components of the vectors correspond to the $x$ - and $y$ directions, respectively. The degree of circular polarization of this optical field is $|\cos (\varphi)|$ and the light is $100 \%$ leftcircularly polarized for $\varphi=0$.
The interaction between the radiation field $\boldsymbol{E}$ and the atoms is given by

$V_{\mathrm{AL}}=-\boldsymbol{E} \boldsymbol{d}$,

where $\boldsymbol{d}$ is the electric dipole operator. Because of the large homogeneous linewidth $(10 \mathrm{~nm})$ of the $D_{1}$-transition of Cs in solid $\mathrm{He}$ and the relatively low laser intensities used, excited state populations and stimulated emission processes can be omitted. As a consequence the atomic evolution under the influence of optical pumping can described by pure ground state equations only [9]. A further consequence of the large optical linewidth is the fact that all four hyperfine transitions of the $D_{1}$-transition are excited simultaneously. As shown earlier $[3,4]$, the creation of spin polarization in Cs trapped in b.c.c. proceeds via repopulation pumping in which the spinpolarization in the excited state is preserved during the optical absorption-emission cycle. The operator $D=D_{\mathrm{ge}}+D_{\mathrm{eg}}$ is defined with

$D_{\mathrm{eg}}=\frac{-1}{2 \hbar} P_{\mathrm{e}} \mathcal{E} \cdot \boldsymbol{d} P_{\mathrm{g}}$ and $D_{\mathrm{ge}}=D_{\mathrm{eg}}^{\dagger}$,

where $P_{\mathrm{g}}=\sum_{\mu}|\mu\rangle\langle\mu|$ and $P_{\mathrm{e}}=\sum_{m}|m\rangle\langle m|$ are projection operators onto ground and excited states respectively. The sums $\sum_{\mu}$ and $\sum_{m}$ extend over all magnetic substates of the $6 S_{1 / 2}$ ground state and the $6 P_{1 / 2}$ excited state. It is shown in the appendix that the rate of change of the ground state density matrix elements under the influence of resonant optical pumping is governed by

$$
\begin{aligned}
(\varrho)_{\mathrm{op}}= & -\frac{2}{\gamma}\left[D_{\mathrm{ge}} D_{\mathrm{eg}}, \varrho\right] \\
& +\frac{3}{\gamma} \sum_{q=-1}^{1}\left(C_{-q}^{1}\right)_{\mathrm{ge}} D_{\mathrm{eg} \varrho} D_{\mathrm{ge}}\left(C_{q}^{1}\right)_{\mathrm{eg}},
\end{aligned}
$$

where $C_{q}^{1}=\sqrt{4 \pi / 3} Y_{1 q}$ is the spherical unity operator and $\gamma$ the homogenous optical linewidth. The two terms in (10) describe depopulation and repopulation pumping respectively. By applying the Wigner-Eckart theorem to the matrix elements of $\boldsymbol{d}$ one sees that the elements of (10) are proportional to the pump rate $\gamma_{p}$, defined as

$\gamma_{\mathrm{p}}=\frac{E_{0}^{2}}{\hbar^{2} \gamma}\left|\left\langle 6 S_{1 / 2}\|d\| 6 P_{1 / 2}\right\rangle\right|^{2}$.

In the experiment fluorescence of the Cs atoms is detected and the fluorescence rate $\mathcal{F}$ (see appendix) is given by

$\mathcal{F}=\mathscr{F}(\varrho)=\frac{4}{\gamma} \operatorname{tr}\left(D_{\text {eg }} \varrho D_{\text {ge }}\right)$

Note that $\mathcal{F}$ is proportional to $\gamma_{\mathrm{p}}$. Alternatively, $\mathcal{F}$ can be expressed as

$\mathcal{F}=\mathcal{F}_{\text {unpol }}\left(1-P_{z} \cos \varphi\right)$,

where $P_{z}=2\left\langle J_{z}\right\rangle$ is the spin polarization and $\mathcal{F}_{\text {unpol }}$ is the fluorescence rate of the unpolarized atomic ensemble [4] in thermal equilibrium. Optical pumping produces a spin polarization $P_{z}^{\max }=2\left\langle J_{z}\right\rangle_{\max }$ which depends on the light intensity with a corresponding minimum in fluorescence $\mathcal{F}_{\text {pol }}$. The 
magnetic resonance process alters $P_{z}$ in a resonant way, which is reflected by a corresponding resonant change in the fluorescence rate $\mathcal{F}$.

\subsection{Relaxation}

As mentioned in the introduction, the electronic spin polarization of alkali atoms embedded in the b.c.c. phase of solid helium reaches values of the order of 1 second. This is a consequence of the spherical symmetry of the local trapping site (spherical bubble) and the non-magnetic properties of the host matrix atoms, as helium has neither an electronic nor a nuclear magnetic moment. The most likely mechanism responsible for the perturbation of the alkali spins are helium lattice vibrations, and more specifically, shape fluctuations of the bubble interface boundary. The atoms of the first solvation shell undergo oscillations around their equilibrium positions and the fluctuating shape of the interface (described by the bubble radius $R(\theta, \phi)$ ) formed by these atoms may be decomposed into spherical harmonics $Y_{k, q}$ as

$R(\theta, \phi, t)=R_{0}+\sum_{k, q} a_{k, q}(t) Y_{k, q}(\theta, \phi)$,

where the coefficients $a_{k, q}(t)$ describe the time dependent fluctuations of a given multipole shape. Without such fluctuations the bubble shape is spherical $R(\theta, \phi, t)=R_{0}$ and the alkali ground state preserves its $S$-state character. The bubble shape fluctuations deform the electronic wave function of the alkali atom, which, in quantum mechanical terms corresponds to an L-mixing interaction. This mixture of higher orbital momentum states, together with spin-orbit and hyperfine interactions in the cesium atom then constitutes a mechanism by which the helium matrix atoms can couple to the alkali spins. For a spin perturbing interaction of a defined multipolarity $k$ the perturbation $H_{f l}(t)$ between the bubble shape and the alkali spins can then be written as [10]

$H_{f l}(t)=\sum_{q=-k}^{k}(-1)^{q} F_{-q}^{k}(t) T_{q}^{k}(S, I)$,

where the $T_{q}^{k}(S, I)$ are spherical tensor operators acting in the combined electronic $(S)$ and nuclear spin $(I)$ spaces that describe atomic properties. The coefficients $F_{q}^{k}(t)$ are determined by the amplitudes $a_{k, q}(t)$ of the bubble oscillations and describe the induced fields coupling to the atom.

The lowest order multipole oscillation is the monopole (or breathing mode) oscillation described by $a_{0,0}(t)$. Because of its scalar nature it will not affect spin coherences nor level populations. However, as shown previously [11], it does affect magnetic hyperfine transitions and hence broadens the corresponding resonance via a modulation of the Fermi contact term of the ground state hyperfine interaction. The next multipole oscillation is the dipole oscillation $(k=1)$. A small (compared to the bubble radius) amplitude dipole deformation of the bubble is equivalent to a small amplitude displacement of the atom from its equilibrium position inside a spherical bubble. The discussion in the following is restricted to dipole $(k=1)$, and quadrupole $(k=2)$, shape oscillations. The bubble-spin interaction can have an electric and/or a magnetic charac- ter. As the atoms have no static electric vector property, the $k=1$ dipole oscillations and the perturbation operator in this case will be dominated by the coupling of induced fluctuating magnetic fields $F_{q}^{1}(t) \propto B_{q}(t)$ to the atomic magnetic moment $T_{q}^{1}(S, I) \propto \mu_{q}=\left(-g_{s} S_{q}+g_{I} I_{q}\right) \frac{\mu_{B}}{h}$. As $g_{I} \ll g_{S}$ contributions from the nuclear moment will be neglected in the calculations, so that the tensor components $T_{q}^{1}(S)$ act in electronic spin space only. In the case of quadrupolar shape oscillations, on the other hand, the second rank tensors $T_{q}^{2}(S, I)$ can not couple to the electronic angular momentum, as the WignerEckart theorem implies that $\left\langle J=1 / 2\left\|T^{2}\right\| J=1 / 2\right\rangle=0$ so that the $k=2$ interaction operators $T_{q}^{2}(I)$ act in nuclear spin space only. Here the simplest interaction will be the coupling of fluctuating electric field gradients, described by $F_{q}^{2}(t)$ to the components of the electric quadrupole moment of the Cs nucleus, described by $T_{q}^{2}(I)$.

The ability of $H_{f l}(t)$ to drive ground state transitions and/or to dephase spin coherences is determined by the magnitude of the power spectral density $J(\omega)$ of the fluctuating field components $F_{q}^{k}(t)$ in the vicinity of the relevant atomic transition frequency. $J(\omega)$ can be calculated according to the Wiener-Khinchine theorem as the Fourier cosine transform of the autocorrelation function $g(\tau)$ of $F_{q}^{k}(t)$ [12]. It is assumed that the fluctuations are isotropic and have an exponentially decaying autocorrelation function

$g(\tau) \equiv\left\langle F_{q}^{k}(t) F_{q^{\prime}}^{k}(t+\tau)^{*}\right\rangle_{t}=\delta_{q, q^{\prime}} \frac{(-1)^{q} f^{2}}{2 k+1} \mathrm{e}^{-|\tau| / \tau_{\mathrm{c}}}$,

where $\tau_{\mathrm{c}}$ is the correlation time and $f$ is the root mean square amplitude of the fluctuation. The power spectrum is then proportional to

$J(\omega) \propto \frac{\tau_{\mathrm{c}}}{1+\omega^{2} \tau_{\mathrm{c}}^{2}}$.

Under the assumption, that in the case under discussion, the fluctuations are determined by He lattice vibrations, $1 / \tau_{\mathrm{c}}$ will be on the order of the Debye frequency $\left(10^{11} \mathrm{~s}^{-1}\right)$ in the b.c.c. phase of solid helium[13]. In this case $\omega \ll 1 / \tau_{\mathrm{c}}$ holds for both hyperfine $(\mathrm{GHz})$ and Zeeman transitions $(\mathrm{kHz})$ in the $6 S_{1 / 2}$ ground state. As a consequence $J(\omega)$ is approximately constant for all these transitions (white noise regime). This assumption is well confirmed by the earlier observation that the longitudinal electronic spin relaxation rate $\gamma_{1}$ has a constant value of approximately $1 \mathrm{~s}^{-1}$ for Larmor frequencies ranging from $70 \mathrm{~Hz}$ to $4 \mathrm{MHz}[5,14]$. The existence of additional noise sources, as, e.g. acoustic vibrations with considerably longer correlation times for which $\omega \tau_{\mathrm{c}} \gg 1$ in the range of investigated frequencies can not be ruled out a priori. As the power density of these fluctuating fields $J(\omega) \rightarrow 0$ they are not able to drive sublevel transitions (population transfers), but contribute nonetheless to spin dephasing [10]. Because of the above mentioned independence of $\gamma_{1}$ on $\omega$ the case of $\omega$ being comparable to the fluctuation rate $1 / \tau_{\mathrm{c}}$ can be ruled out, so that in the following the problem will be treated for the two extreme cases $\omega \ll 1 / \tau_{\mathrm{c}}$, and $\omega \gg 1 / \tau_{\mathrm{c}}$ only.

In order to obtain an expression for the relaxation term $(\dot{Q})_{\text {rel }}$ of the Liouville equation the relaxation mechanism has been treated as a Markovian process following the methods described, e.g., in $[12,15,16]$. Only the main formulas are 
presented without discussing details. In the secular approximation $(\dot{\varrho})_{\text {rel }}$ can be written in a compact operator form (see Chapt. VIII, (42) in [12]) as

$(\grave{\varrho})_{\mathrm{rel}}=-\frac{1}{2 \hbar^{2}} \sum_{q, p}(-1)^{q} J\left(\omega_{p}^{q}\right)\left[\left(T_{-q, p}^{k},\left[T_{q, p}^{k}, \varrho\right]\right]\right.$,

where the operators $T_{q, p}^{k}$ are defined as expansion coefficients of the time dependence of the $T_{q}^{k}$ according to

$\mathrm{e}^{i H_{0} t / \hbar} T_{q}^{k} \mathrm{e}^{-i H_{0} t / \hbar}=\sum_{\mathrm{p}} T_{q, p}^{k} \mathrm{e}^{i \omega_{\mathrm{p}}^{q} t}$

in the interaction picture. In the special case of non equidistant Zeeman levels $|(F), M\rangle$ the summation index $p$ represents any combination of quantum numbers $p=\left(F, M ; F^{\prime}, M^{\prime}\right)$ and one has

$T_{q, p}^{k}=\left\langle\left(F^{\prime}\right), M^{\prime}\left|T_{q}^{k}\right|(F), M\right\rangle\left|\left(F^{\prime}\right), M^{\prime}\right\rangle\langle(F), M|$

and

$\omega_{\mathrm{p}}^{q}=\omega_{\mathrm{p}}=\omega_{F^{\prime}, M^{\prime}}-\omega_{F, M}$,

where the $\omega_{F, M}$ are the eigenfrequencies of $H_{0}$. Since $J(\omega)$ has been assumed to be constant it can be taken out of the sum. By applying the Wigner-Eckart theorem to the operators $T_{q}^{k}$ one sees that (13) is proportional to the rate

$\gamma=J(\omega)\left|\left\langle K\left\|T^{k}\right\| K\right\rangle\right|^{2}$,

where $\left\langle K\left\|T^{k}\right\| K\right\rangle$ is the reduced matrix element of $T^{k}(K)$ with $K=S$ for $k=1$ and $K=I$ for $k=2$ respectively. This relaxation rate $\gamma$ will be used as a parameter in the numerical calculations.

When the light field is switched off $\varrho$ relaxes towards the thermal equilibrium value

$\varrho_{0}=\sum_{F, M} \varrho_{0}^{F}|(F), M\rangle\langle(F), M|$,

in which the relative populations of the two hyperfine ground states is determined by the Boltzmann factors $b_{F}=$ $\exp \left(-\hbar \omega_{F} / k_{B} T\right)$. For a normalized total ground state population the thermal steady-state density matrices of the two hyperfine levels $F=3,4$ are given by $\varrho_{0}^{F=3}=1 /(7+9 b)$ and $\varrho_{0}^{F=4}=b /(7+9 b)$ where $b=b_{4} / b_{3}=\exp \left(-\hbar \omega_{h f_{s}} / k_{B} T\right)$. A rigorous treatment of the relaxation towards this thermal equilibrium distribution $\varrho_{0}$ would require a quantum mechanical treatment of the helium lattice (heat reservoir) in the Hamiltonian of (12). In the calculations, (13) was corrected by appropriate Boltzmann factors, so that its steady-state solutions yield the correct thermal populations of the hyperfine levels $[12,15]$. The population differences due to the Zeeman shifts inside each hyperfine multiplet $F$ can be neglected at the temperature and field, at which the experiments were performed.

\section{5}

\section{Relaxation of multi-quantum coherences}

Algebraic expressions were derived relating the $N$-quantum coherence rates $\gamma_{N+1}$ to the rate $\gamma$ introduced above. Only transitions starting from the state $|(4), 4\rangle$ are considered, since the optical pumping process mainly populates this state. Evaluation of the right hand side of (13) then shows that the decay of the coherences is described by uncoupled differential equations of the form

$\left(\dot{\varrho}_{M, M^{\prime}}\right)_{\mathrm{rel}}=-\gamma_{M, M^{\prime}} \varrho_{M, M^{\prime}}$.

The intrinsic relaxation rate $\gamma_{N+1}$ of the $N$-photon coherence $\langle(4), 4-N|\varrho|(4), 4\rangle$ in (3) is defined as $\gamma_{N+1} \equiv \gamma_{4,4-N}$. Table 1 shows the analytical results of this calculation for the cases of relaxation considered here. The same results are displayed as ratios $\gamma_{N+1} / \gamma_{2}$ in Fig. 3. It can be seen that for relaxations dominated by the $T^{1}(S)$ perturbation, the relaxation rates grow monotonically with $N$ (linearly in the limit $\omega \tau_{\mathrm{c}} \ll 1$ and quadratically in the limit $\left.\omega \tau_{\mathrm{c}} \gg 1\right)$, while for the quadrupole relaxation mechanism $T^{2}(I)$ there is a maximum in the relaxation rates and in the limit $\omega \tau_{\mathrm{c}} \ll 1$. The 8-photon coherence has even a slower relaxation than the one-photon coherence.

\subsection{Gain in spectroscopic sensitivity}

It is now possible to estimate the gain of spectroscopic sensitivity of the $\mathrm{N}$-photon resonances compared to the $N=1$ single photon resonance for different relaxation

\begin{tabular}{lcc}
\hline & $T^{1}(S)$ & $T^{2}(I)$ \\
\hline$\omega \tau_{c} \ll 1$ & $\frac{N+8}{36}$ & $\frac{1}{15}\left(1-\frac{3}{5}\left(\frac{N-4}{4}\right)^{2}\right)$ \\
$\omega \tau_{c} \gg 1$ & $\frac{1}{288} N^{2}$ & $\frac{3}{175}\left(2-\frac{1}{4}(N-4)^{2}+\frac{(N-4)^{4}}{128}\right)$ \\
\hline
\end{tabular}

TABLE $1 \quad \gamma_{N+1} \equiv \gamma_{4,4-N}$ in units of $\gamma$ for the four cases of relaxation under discussion
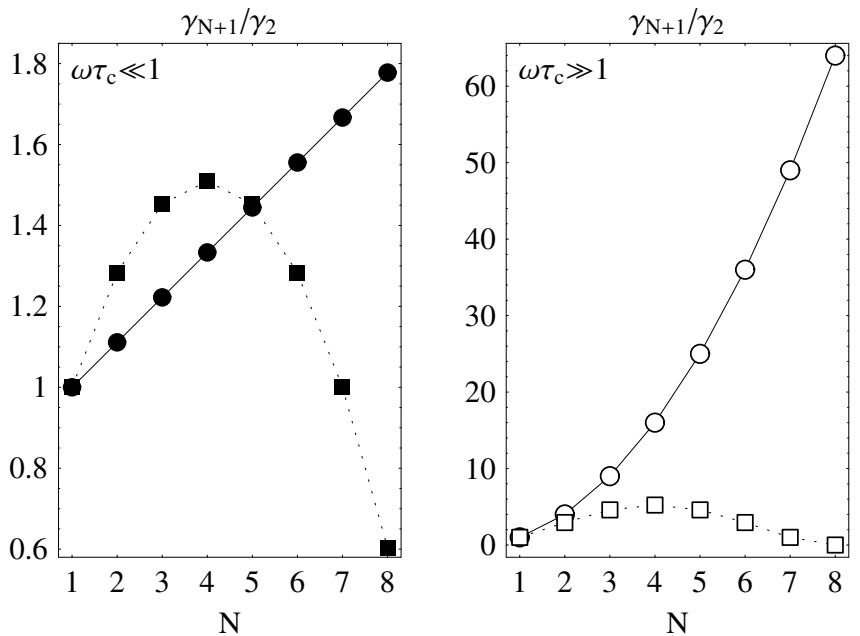

FIGURE $3 \gamma_{N+1} / \gamma_{2}$ for the four relaxation mechanism under discussion. $\gamma_{N+1} \equiv \gamma_{4,4-N}$ is defined as the relaxation rate of the $\varrho_{4,4-N}$ coherence. $\omega \tau_{\mathrm{c}} \ll 1: T^{1}(S)$ (filled circles) and $T^{2}(I)$ (filled squares); $\omega \tau_{\mathrm{c}} \gg 1: T^{1}(S)$ (open circles) and $T^{2}(I)$ (open squares). Corresponding points are joined by lines to guide the eye 


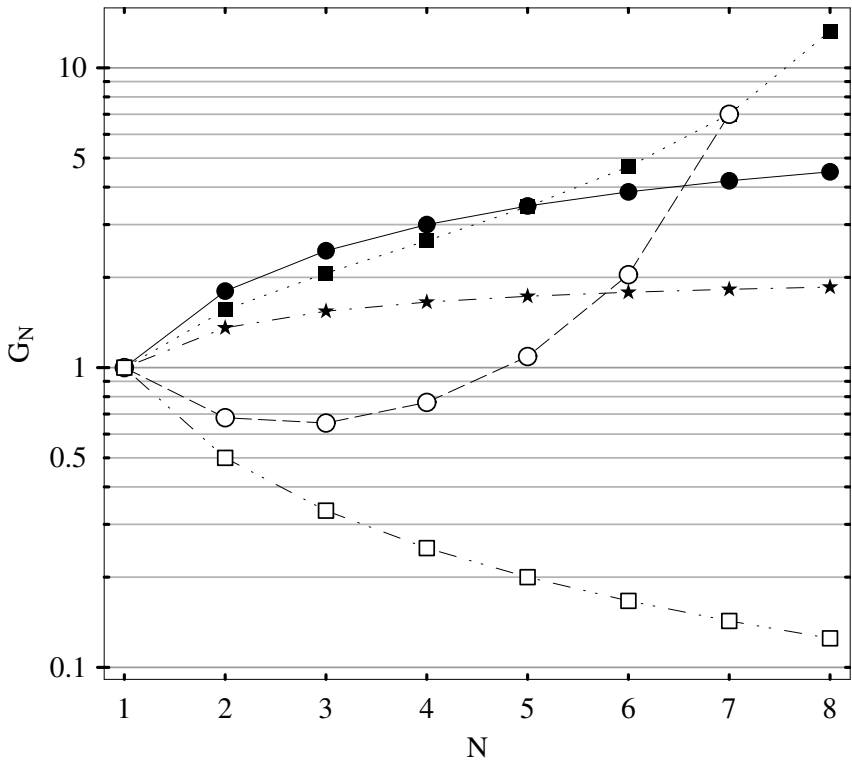

FIGURE 4 Estimations for the gain in spectroscopic sensitivity $G_{N}$ of the $N$-photon resonances with respect to the single photon resonance for several multipole relaxation mechanisms and optical pumping relaxation: For $\omega \tau_{\mathrm{c}} \ll$ $1: T^{1}(S)$ (filled circles) and, $T^{2}(I)$ (filled squares); for $\omega \tau_{\mathrm{c}} \gg 1: T^{1}(S)$ (open circles) and, $T^{2}(I)$ (open squares); and for the relaxation by optical pumping only (stars). Corresponding points are joined by lines to guide the eye. The values are calculated from (5) in Sect. 2.2

schemes. As in section Sect. 2.2 it is assumed that under optimal conditions the amplitudes of the multi-photon resonances in each generation are independent of $N$, so that the gain is basically determined by the ratio of linewidths $G_{N}=\gamma_{2} / \gamma_{N+1} N$ defined in (5). Figure 4 shows the resulting values of $G_{N}$ for the four relaxation processes.

The gain factor $G_{N}$ has also been calculated for the simplest case of relaxation, in which the decay of the coherences is due to optical pumping only. The relaxation rates $\gamma_{N+1}$ for that case are obtained from (10). Optical power broadening was the only relaxation mechanism considered in the work by Pazgalev and Alexandrov [2]. From Fig. 4 it can be seen that $G_{N}$ strongly depends on the underlying relaxation mechanism of the spin ensemble. For a pure dipolar dephasing relaxation mechanism described by $T^{1}(S)$ in the limit $\omega \tau_{\mathrm{c}} \gg 1$ one even finds a strong loss in sensitivity, whereas the highest gain is predicted for a quadrupolar relaxation mechanism described by $T^{2}(I)$ in the limit $\omega \tau_{\mathrm{c}} \ll 1$. It is also interesting to note that in the case of quadrupolar relaxation in the limit $\omega \tau_{\mathrm{c}} \gg 1$ the calculation shows that $\gamma_{9}=0$, i.e. that the $N=8$-photon coherence between the states $M=4$ and $M^{\prime}=-4$ is not effected by the relaxation interaction.

It is interesting to further note that only a factor of approximately two can be won in resolution by using multiphoton resonances when the decay of spin coherence is dominated by optical pumping.

The data given in the figure can only serve as a rough estimate, as they merely consider magnetic resonance transitions originating from the $|(4), 4\rangle$ state and as they rely on the validity of the algebraic formulas given in Sect. 2.2. The complex steady state population distribution among the ground states resulting from the interplay of magnetic and optical interactions has not been considered. A more realistic estimation of
$G_{N}=R_{N} / R_{1}$ can only be achieved by complete calculations of the ODMR spectra from the solutions of the steady state master equation (6) for realistic parameters. The results of such calculations will be presented below.

\section{7}

\section{Technical details of the calculations}

The multi-photon ODMR spectra of Cs has been calculated numerically by solving (6) for the density matrix $\varrho$ describing the 16 sublevels of the $6 S_{1 / 2}$ ground state hyperfine structure. Since all coherences between the two hyperfine levels $F=3$ and $F=4$ are neglected this calculation is equivalent to solving 130 coupled linear algebraic equations. From $\varrho$ the ODMR signal $\mathcal{F}(\varrho)$ could then be obtained from (11). The system of equations was first formulated in a symbolical way using Mathematica [17] and then solved, after replacing all parameters by appropriate values, by a spare-matrix solving algorithm, implemented in Mathematica. An important task consisted in determining from the rather complicated ODMR spectra the amplitudes and widths of single peaks. For this purpose the system of equations were differentiated algebraically with respect to the radio frequency $v_{\mathrm{rf}}$. After inserting the previously obtained steady-state solutions for the elements of $\varrho$, a new system of 130 equations is obtained, whose solutions yield $\partial \varrho / \partial \nu_{\mathrm{rf}}$. Here the interchangeability of the differentiations with respect to time and frequency in the master equations was used. Since $\mathcal{F}$ is linear in $\varrho$ the derivative of the fluorescence rate with respect to the $\mathrm{rf}$ frequency is given by $\partial \mathcal{F} / \partial v_{\mathrm{rf}}=\mathcal{F}\left(\partial \varrho / \partial \nu_{\mathrm{rf}}\right)$. By repeating this procedure higher derivatives of $\mathcal{F}$ with respect to the frequency can be obtained. From the first two derivatives the amplitudes and widths of the Lorentzians are easily found, if the underlying background can be regarded as locally flat. This condition is well fulfilled for the non saturated $N$-photon peaks sitting on top of power broadened lower order peaks.

\section{$4 \quad$ Experiment \\ 4.1 Experimental setup and data recording}

The experimental procedure used is an improved version of the one described previously [1]. Only the main features and the modifications will be addressed here. The experiments were performed on Cs atoms implanted in a helium crystal contained in a pressure cell immersed in a superfluid helium bath cooled by pumping on the bath (Fig. 5). Quartz windows provide optical access from three orthogonal directions. The cesium atoms are implanted into the crystal from a metal target by means of laser ablation. The temperature of the crystal is actively stabilized to $1.5 \mathrm{~K}$ at a level of $10^{-5} \mathrm{~K}$ over intervals of 100 seconds [18].

The cesium atoms are excited on the $D_{1}$ transition $\left(6 S_{1 / 2} \rightarrow 6 P_{1 / 2}\right)$ at $850 \mathrm{~nm}$ with a beam from a single-mode extended cavity diode laser. The absorption is monitored by detecting the $6 P_{1 / 2} \rightarrow 6 S_{1 / 2}$ fluorescence light at $888 \mathrm{~nm}$ using a cooled, biased avalanche-photodiode. An interference filter suppresses scattered laser light. A set of three Helmholtz coils inside the pressure cell allows the application of rf fields in any direction. Three layers of $\mu$-metal surround the cryostat and suppress laboratory magnetic fields by more than three orders of magnitude. 


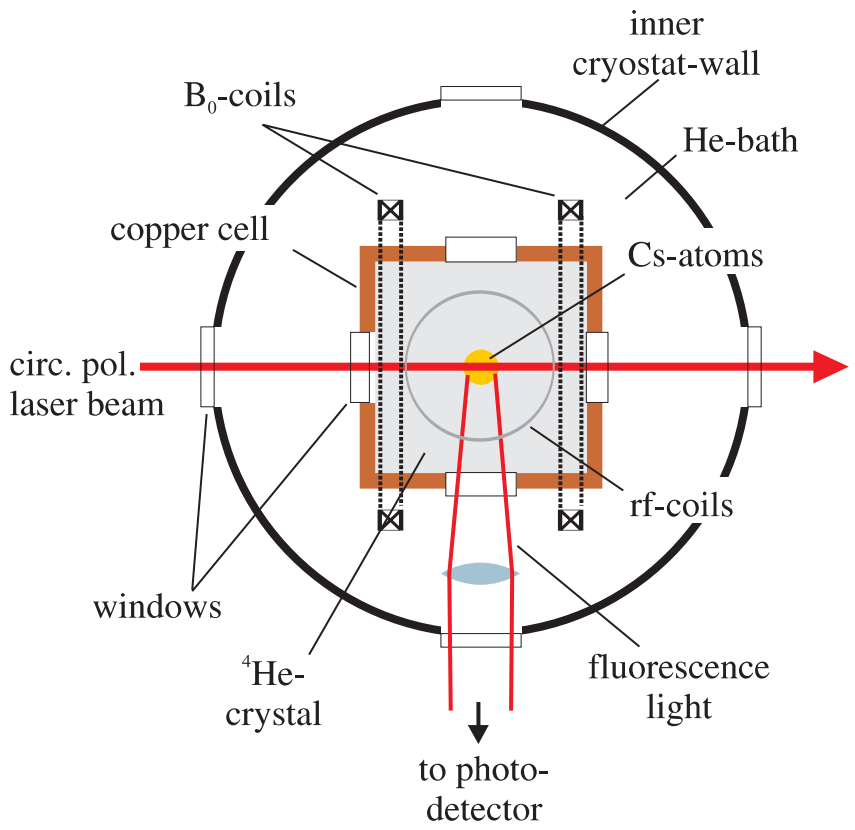

FIGURE 5 Section (top view, not to scale) through the helium containing part of the cryostat. The isolation vacuum part and a liquid nitrogen shield are not shown

A new feature of the set-up is the generation of the $B_{0}$ field by a pair of superconductive Helmholtz coils operated in a self-sustained (persistent) current mode. After injecting a current from an external current source the coils are bypassed by closing a temperature-controlled superswitch (graphite resistor) mounted in an evacuated copper housing placed at the bottom of the helium bath.

The data acquisition was also improved by significantly reducing the scan speed. In the previous experiments spectral distortions due to fast scanning led to significantly asymmetric line shapes. The sweep duration was now increased from $20 \mathrm{~s}$ to $100 \mathrm{~s}$ while reducing at the same time the swept frequency span from $50 \mathrm{kHz}$ to $15 \mathrm{kHz}$. This implied that only multi-photon resonances of the type $|(4), 4\rangle \rightarrow|(4), 4-N\rangle$ in the $F=4$ multiplet were recorded. The non-observation of the lines in the $F=3$ multiplet constitutes nonetheless a minor loss of information, since due to the optical pumping process almost all population is transferred to the $|(4), 4\rangle$ state, so that the $|(3), 3\rangle \rightarrow|(3), 3-N\rangle$ transitions are in general much weaker and carry no relevant additional information.

\section{2}

\section{Experimental results}

Figure 6 shows a series of eight ODMR-spectra taken in the b.c.c. phase of solid ${ }^{4} \mathrm{He}$ at a temperature of $1.501 \mathrm{~K}$ and a pressure of $26.71 \mathrm{bar}$. The spectra were recorded in a field of $1.0414 \mathrm{mT}$ by scanning the frequency of the $\mathrm{rf}$ field over the multiphoton resonances of the $F=4$ multiplet. The origin of the frequency axis corresponds to the frequency of the $|(4), 4\rangle \rightarrow|(4), 3\rangle$ one-photon transition $\left(v_{\text {ref }}=3633.72 \mathrm{kHz}\right)$. The spectra were recorded for different rf powers, chosen experimentally such that the multi-photon lines of a given order $N$ present the dominant feature of the spectrum. The corresponding relative rf field strengths

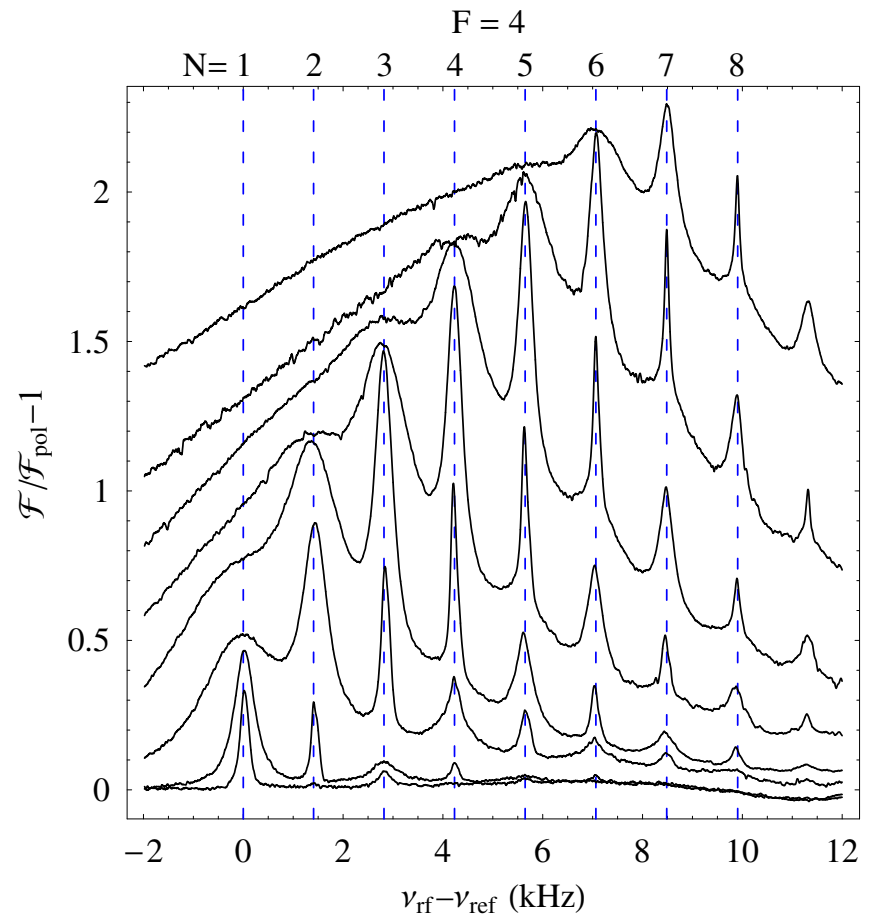

FIGURE 6 Background corrected (see the text), measured multiphoton resonance spectra in a field $B_{0}$ of $1.0414 \mathrm{mT}$. The origin of the frequency axis corresponds to the frequency of the $|(4), 4\rangle \rightarrow|(4), 3\rangle$ one-photon transition $\left(v_{\text {ref }}=3633.72 \mathrm{kHz}\right)$. Dashed lines indicate the positions of the $N$-photon resonances $|(4), 4\rangle \rightarrow|(4), 4-N\rangle$. The rf power is increased (ratios given in the text) in each of the eight spectra going from bottom to top in such a way that the newly appearing $N$-photon lines show an optimal spectral resolution

for the subsequent spectra (top to bottom in Fig. 6) were $10000: 6662: 4436: 2826: 1669: 933: 192: 44$.

The extension of the scan time is however accompanied by a diminished signal to noise ratio. In the raw data of the presented measurements the multiphoton spectra are superimposed on a monotonously decreasing fluorescence signal, which is due to a loss of atoms by recombination of the implanted Cs atoms during the rf sweep with clusters and/or other Cs atoms. Before each sweep these clusters were dissociated by applying a Nd: YAG-laser pulse focused into the Cs doped volume. The atomic signal then drops on a time scale of several tens of seconds, which implies that the signal to noise ratio of the data points steadily drops as the scan proceeds. Hence the data points show a steadily decreasing signal to noise ratio during a sweep. The ordinate of the spectra presented in Fig. 6 represents $\mathcal{F} / \mathcal{F}_{\mathrm{b}}-1$, where $\mathcal{F}$ is the recorded fluorescence and $\mathcal{F}_{\mathrm{b}}$ the background fluorescence underlying the magnetic resonance spectra proper. $\mathcal{F}_{\mathrm{b}}$ was determined by fitting an appropriate function to the time dependent background. In this way the normalization $\mathcal{F} / \mathcal{F}_{\mathrm{b}}-1$ does not only remove the background, but it also corrects for the loss of atoms and hence for the apparent loss of signal amplitude during the scan. The rf frequency was swept from higher to lower frequencies, which explains the larger noise levels at low rf frequencies in Fig. 6. This procedure of normalizing the signals has the further advantage that the experimental signal amplitudes can be compared to the corresponding theoretical spectra on an absolute scale. 


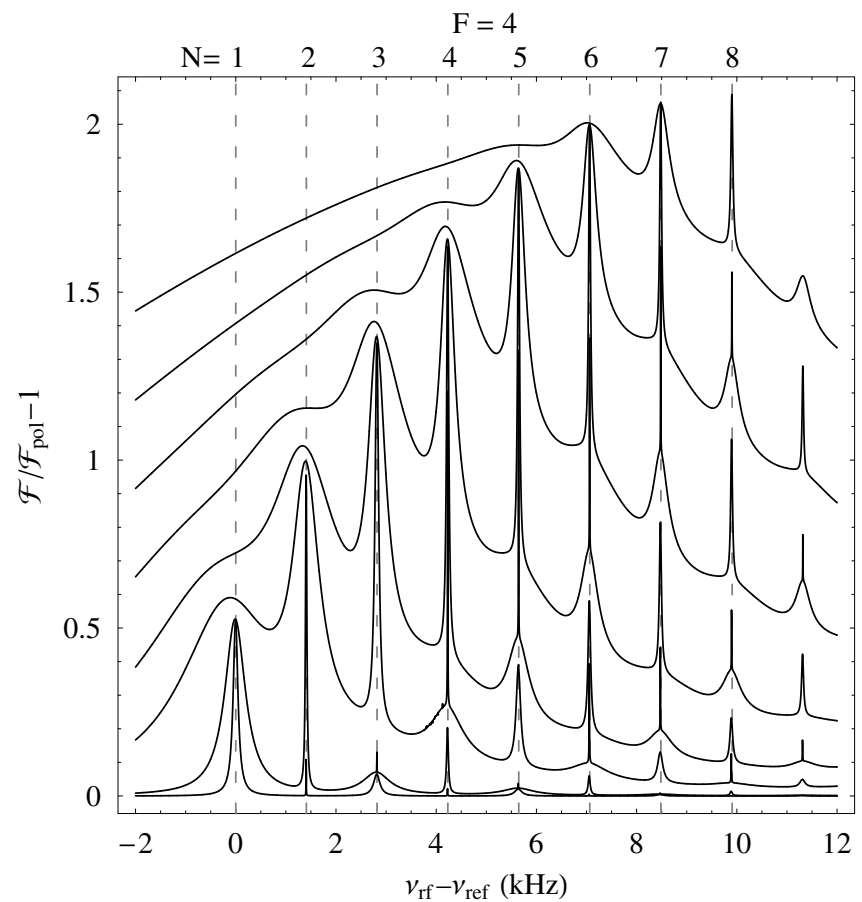

FIGURE 7 Multi-photon spectra calculated by solving master equation (6) for various values of $B_{\mathrm{rf}}$. The fixed parameters $B_{0}, \gamma_{\mathrm{p}}$, and $\varphi$ were adjusted to the experimental values. $B_{\mathrm{rf}}$ was changed in the same proportions as in the experiment shown in Fig. 6. No intrinsic relaxation was considered and the linewidths are determined by rf and optical pumping broadening only. The fluorescence rate $\mathcal{F}$ is represented in units of the thermal equilibrium fluorescence rate $\mathcal{F}_{0}$ of the unpolarized spin ensemble

\section{3}

\section{Comparison with the calculations}

The experimental data was reproduced by calculating the ODMR spectra according to the method given in Sect. 3.1. Since the linewidths of the measured resonances were still dominated by the technical and optical pumping broadening rather than by an intrinsic relaxation processes, the later could be neglected, by setting $\gamma=0$.

Beside $B_{\text {rf }}$ which is different for all eight spectra, three common parameters: $B_{0}, \gamma_{\mathrm{p}}$, and the parameter $\varphi$ characterizing the degree of circular polarization, entered the calculations. By fitting Lorentzians to the measured multi-photon spectra (Fig. 6) $B_{0}$ was inferred from the line positions in accordance with the Breit-Rabi formula taking the perturbation of the hyperfine constant by the helium matrix into account [1]. A typical value for $\varphi$ of $0.5 \mathrm{rad}$ could be obtained from earlier measurements [14]. From the measured light intensity of $78 \mathrm{~mW} / \mathrm{cm}^{2}$ the optical pumping rate was inferred to be $\gamma_{\mathrm{p}} \approx 390 \mathrm{~s}^{-1}$ using again results of recent experiments [4]. Figure 7 shows the calculated ODMR spectra. By representing the quantity $\mathcal{F} / \mathcal{F}_{\text {pol }}-1$ the theoretical results are commensurable with the experimental spectra of Fig. 6 on an absolute scale.

Since there is no precise calibration of the rf field inside the pressure cell, $B_{\text {rf }}$ was adjusted for the uppermost curve in Fig. 7 so that optimal consistency was achieved with the corresponding measured spectrum. This was at $B_{\mathrm{rf}}=2.57 \mu \mathrm{T}$, which is in good agreement with the value estimated from the output current of the rf generator and the dimensions of the rf field coils. The other curves were then obtained at lower rf

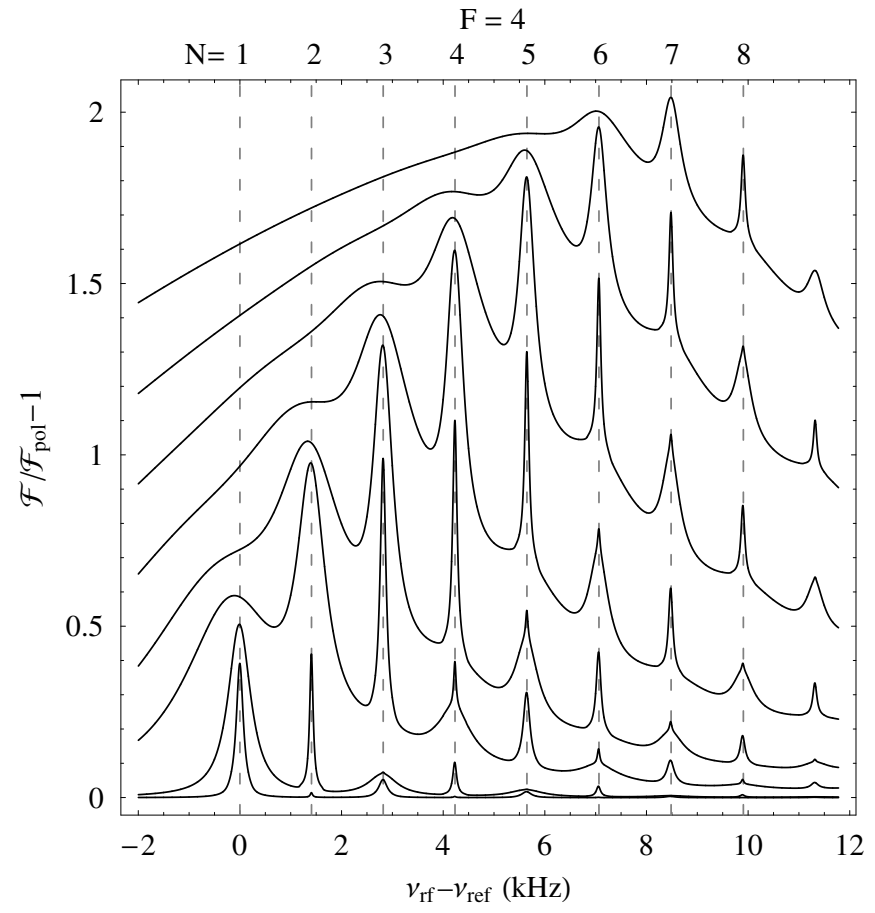

FIGURE 8 The calculated data of Fig. 7 convoluted with a Loretzian shaped curve with a FWHM of $70 \mathrm{~Hz}$. The convolution mimics the influence of the technical broadening which is is not included in the master equation calculation leading to the results shown in Fig. 7

fields, with amplitudes in the same ratios as in the experiment. All other parameters were kept fixed.

The peaks of the calculated spectra whose widths are determined by rf-power broadening reproduce closely the overall structure of the experimental data with respect to their relative heights and shapes, whereas the lesser broadened peaks are too high in amplitude and too small in width. As this is assigned to small magnetic field inhomogeneities in the experiment, such inhomogeneities are taken into account in the calculations by convoluting the theoretical data with a Lorentzian distribution of the magnetic fields around the average field value. A FWHM of $70 \mathrm{~Hz}$ of the convolution profile (corresponding to a field inhomogeneity of $2 \times 10^{-5}$ ) yielded the best agreement between measurement and theory. Not only is the agreement excellent regarding the different relative heights and widths of the peaks, but the amplitudes of the spectra also coincide on an absolute scale. It must be stressed again that a single set of parameters was used for all spectra. A small residual discrepancy of the absolute signals may be assigned to stray fluorescence light of non-atomic origin and/or to electronic offsets, which both introduce errors in the normalization procedure of the experimental signals. Compared to the previous study [1], details of the calculated spectra, such as the reduced $N=2$-photon peak height in the second (from the bottom) spectrum fit the experimental data well, thus confirming the suitability of the model.

\section{Effect of intrinsic multipole relaxations on the multi-photon spectra}

One of the goals of this work was to extract information about the mechanism which determines the spin relax- 
ation of $\mathrm{Cs}$ atoms in the cubic phase of ${ }^{4} \mathrm{He}$ by comparing experimental multi-photon spectra to theoretical spectra based on different relaxation models. This difficult goal has not yet been reached because the experimental line shapes are dominated by an inhomogeneous technical broadening, whose origin lies most probably in small (effects are on the order of $\Delta B / B \approx 10^{-5}$ ) residual magnetic field inhomogeneities. In future experiments it should be possible to overcome this problem. Nonetheless, in order to demonstrate the anticipated consequences of different relaxation mechanisms the multiphoton spectra are calculated for ideal conditions, i.e. for a perfectly homogeneous magnetic field.

Two cases of dipolar and quadrupolar perturbations are considered, and are described by $T^{1}(S)$, and $T^{2}(I)$, respectively. We restrict the discussion to the limit $\omega \tau_{\mathrm{c}} \ll 1$, for which the fluctuating interactions not only lead to transverse spin relaxation, but also to longitudinal relaxation by inducing sublevel transitions. In this case the parameter $\gamma$, which characterizes the strength of the relaxation mechanism under consideration, can be inferred from the experimentally determined longitudinal relaxation rate $\gamma_{1}$, at which $\left\langle J_{z}\right\rangle$ relaxes. In the limit $\omega \tau_{\mathrm{c}} \gg 1$ only transverse spin relaxation occurs, and no reasonable quantitative predictions of $\gamma$ can be made with present knowledge. In order to determine $\gamma$ from the experimental value of $\gamma_{1}$ it was necessary to solve $\dot{\varrho}=(\dot{\varrho})_{\text {rel }}$ for both cases of multipolarity, assuming $\varrho_{4,4}(t=0)=1$ as the initial condition for the normalized density matrix. From $\varrho(t)$ the temporal evolution of $\left\langle J_{z}\right\rangle$ was obtained.

In the case of $T^{1}(S)$ relaxation it was possible to derive the following algebraic function that describes the decay of $\left\langle J_{z}\right\rangle$

$\left\langle J_{z}\right\rangle(t)=\frac{1}{62}\left(24 \mathrm{e}^{-\frac{4}{9} \gamma t}+7 \mathrm{e}^{-\frac{1}{72} \gamma t}\right)$.

The polarization is thus seen to have two decay channels whose rates differ by a factor of 32 . Moreover, the slow channel is responsible for the decay of $77 \%$ of the initial polarization. The time scales in previous $T_{1}$ measurements were too small to reveal the presence of two strongly differing time scales, and the question arises as to which of the two channels should be identified with the measured values of $\gamma_{1} \approx 1 \mathrm{~s}^{-1}$. As in the experiments, a large amplitude decay of the polarization was observed and it seems natural to identify the experimental $\gamma_{1}$ value with $\frac{4}{9} \gamma$, which yields $\gamma=2.25 \mathrm{~s}^{-1}$. Thus, the second component has a decay time of 32 seconds. This relaxation rate is hard to measure as it is comparable to the loss rate of atoms due to recombination as described in Sect. 4.2. In the case of $T^{2}(I)$ relaxation attempts to derive an algebraic function describing the decay of $\left\langle J_{z}\right\rangle$ did not suceed. However, numerical calculations suggest that $\left\langle J_{z}\right\rangle$ has an exponential decay with a single rate, for which comparison with the experimental decay yields $\gamma \approx 88.3 \mathrm{~s}^{-1}$.

These values of $\gamma$ form the basic input parameters, besides the experimentally determined parameters $B_{0}$ and $\gamma_{\mathrm{p}}$, for the theoretical calculations. As already stated several times the experimental spectra were limited by technical line broadening. The pump rate $\gamma_{\mathrm{p}}$ in the experiments was chosen in such a way that the broadening due to optical pumping was slightly smaller than the technical linewidth of the unsaturated multi-photon resonances. If in future experiments the inhomogeneous broadening can be substan- tially reduced or eliminated, then, of course, the optimal pump rate for the experiments should be reduced correspondingly. For this reason it was decided to calculate the spectra comparing the influence of the perturbation multipolarity for a light intensity, which is 10 times lower than in the experiments described above. Figure 9 and Fig.10 show the

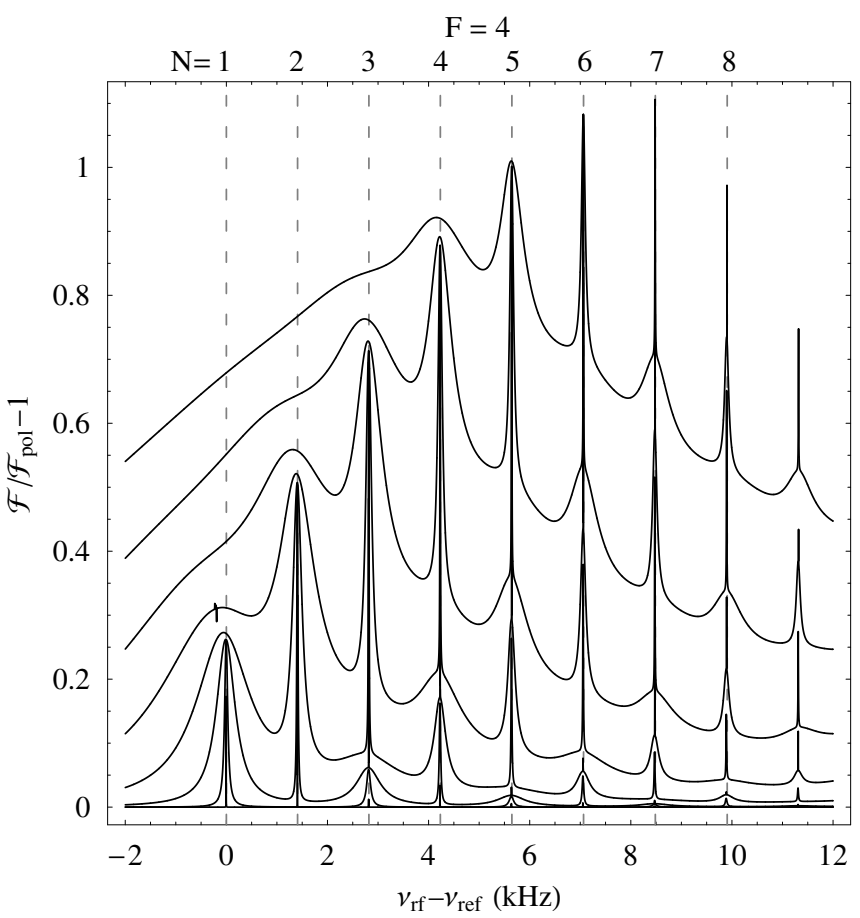

FIGURE 9 Calculated multi-photon spectra for $T^{1}(S)$ relaxation in the limit of $\omega \tau_{\mathrm{c}} \ll 1$. The optical pumping rate $\gamma_{\mathrm{p}}$ is ten times lower than in the experiment

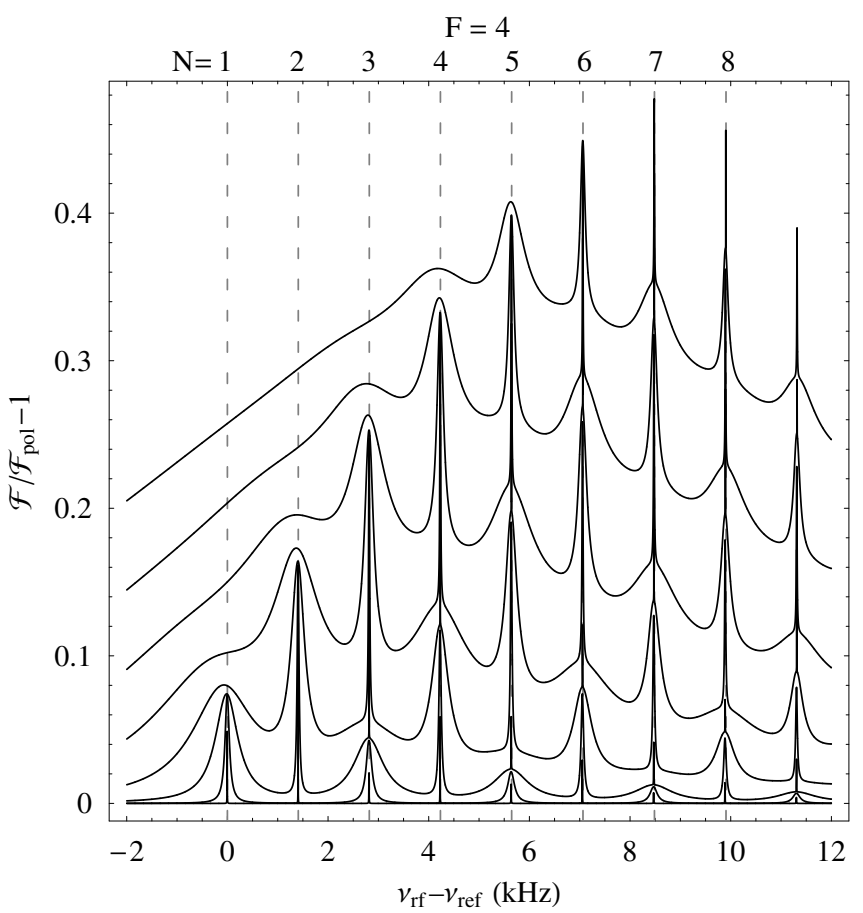

FIGURE 10 Calculated multi-photon spectra for $T^{2}(I)$ relaxation in the limit of $\omega \tau_{\mathrm{c}} \ll 1$. The optical pumping rate $\gamma_{\mathrm{p}}$ is ten times lower than in the experiment 
spectra obtained with $\gamma_{\mathrm{p}}=39 \mathrm{~s}^{-1}$ for $T^{1}(S)$ and $T^{2}(I)$ relaxation respectively. The parameter distinguishing the curves in these figures is the rf field amplitude $B_{\mathrm{rf}}$. For each curve $B_{\mathrm{rf}}$ was chosen such as to maximize the spectroscopic resolution of the multi-photon generation that emerged in that spectrum.

Note that the same spectra were also calculated as in Figs. 9 and 10 with $\gamma_{p}=390 \mathrm{~s}^{-1}$, i.e., the pump rate used in the present experiments. As anticipated the resulting resonance systems look identical for $T^{1}(S)$ and $T^{2}(I)$ relaxation, as the widths are dominated by optical pumping broadening. These spectra are not shown.

The amplitude $A_{N}$ and widths (FWHM) $\Delta v_{N}$ of the dominant non-saturated multi-photon line were determined in each trace of Figs. 9 and 10. The dependencies of the relative amplitudes $A_{N} / A_{N=1}$ and relative inverse widths $\left(1 / \Delta v_{N}\right) /\left(1 / \Delta v_{1}\right)$ on the multi-photon order $N$ are shown in Fig. 11a and $b$.

In the simplified discussion of magnetic resonance in Sects. 2.2 and 3.6 the gain in spectroscopic sensitivity $G_{N}=$ $\Delta v_{1} / \Delta v_{N}=\gamma_{N+1} / \gamma_{2} N$ depended only on the relative line widths. The data in Fig. 11a show the ratios of linewidths from the present complete calculation. Note that the qualitative $N$-dependence is similar to the one shown in Fig. 4 derived from the simplified treatment, which did not consider the optical pumping process by which coherences are also destroyed.

The optical pumping process is also responsible for the fact that the amplitudes have a significant $N$-dependence (Fig. 11b) due to the complex interplay of the production of population differences by optical pumping and their destruction by saturated lower order multi-photon transitions. In the discussion of Sects. 2.2 and 3.6 it was assumed that all populations were in the $|(4), 4\rangle$ state, which implied that the amplitudes for optimal sensitivity were found to be $N$-independent.

As both the amplitudes and the widths of the multi-photon resonances depend on $N$, the gain in spectroscopic resolution has to be calculated according to

$G_{N}=\frac{A_{N}}{\Delta v_{N}} / \frac{A_{1}}{\Delta v_{1}}$
The corresponding dependence is shown in Fig. 11c. For the case of the dipolar $T^{1}(S)$ relaxation the gain grows in a monotonous way to reach a maximum value of approximately 8 for the eight-photon transition. It is interesting to note that this factor corresponds to the naive expectation discussed in the introduction. In the case of quadrupolar $T^{2}(I)$ relaxation a maximum gain of approx. 5.6 is found for the five-photon process and there is a loss of sensitivity for higher order processes.

\section{$6 \quad$ Summary and outlook}

A theoretical analysis of the influence of stochastic spin perturbing interactions of dipolar and quadrupolar symmetry on the spectra of multi-photon transitions in the ground state of cesium in solid helium has been performed. Algebraic results for the multipole relaxation rates were derived for both cases in the limits $\omega \tau_{\mathrm{c}} \gg 1$ and $\omega \tau_{\mathrm{c}} \ll 1$. Numerical results for the multi-photon spectra taking optical pumping, magnetic resonance and relaxation into account were obtained. The results were used to predict the gain in spectroscopic resolution of multi-photon transitions and yielded a maximum gain factor of 8 for quadrupolar relaxation. Unfortunately the quality of the present experimental spectra is not sufficient to discriminate between these two most likely relaxation mechanisms. However, the experimental spectra can be reproduced well by the calculations taking field inhomogeneities into account. In the future it is planned to reduce the magnetic field inhomogeneities by appropriate shim coils, and by replacing recently identified remote parts of the cryostat which show a residual magnetization.

Of course it would be interesting to measure the relaxation rates of the individual multi-quantum coherences directly as their $N$-dependence offers the most promising way to identify the multipolarity of the perturbation (Fig. 11a). Another promising approach to this goal is the recording of free-induction decay signals following the selective excitation of a given resonance. The relative intensities of the singlephoton lines at low rf power, which are barely visible in Figs. 9 and 10 , seem to offer yet another characteristic feature, which reflects the multipolarity of the interactions. Work along these directions is in progress.
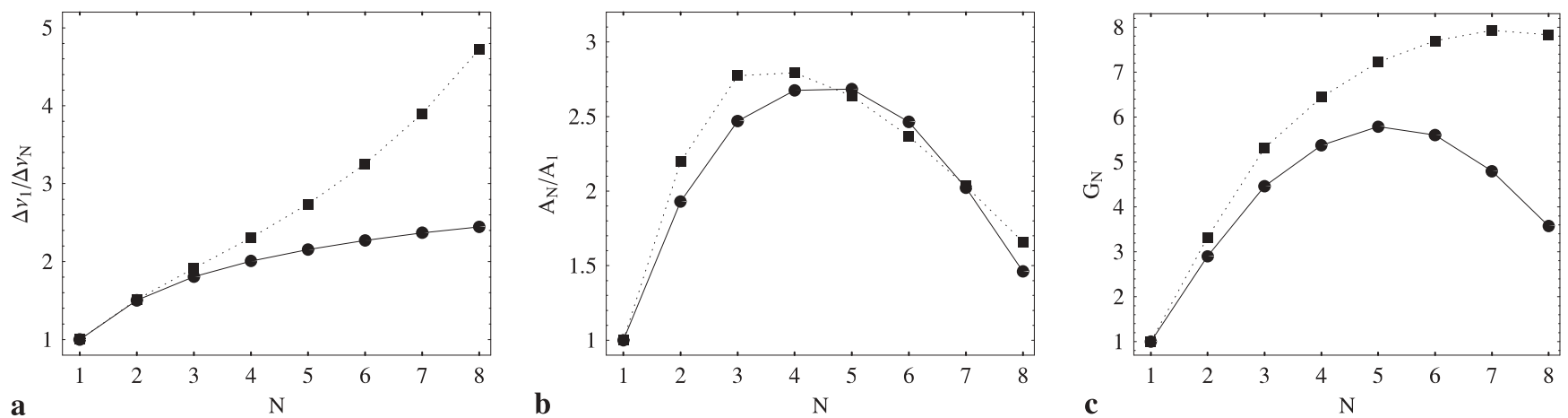

FIGURE 11 a Ratio of inverse linewidths $\frac{1}{v_{N}} / \frac{1}{v_{1}}$ determined from the spectra of Figs. 9 and 10. Filled circles: $T^{1}(S)$-relaxation, filled squares: $T^{2}(I)$ relaxation. b Ratio of amplitudes $A_{N} / A_{1}$. c Gain of spectroscopic resolution $G_{N}$. Note that $G_{N}$ can be calculated by multiplying the corresponding data in $\mathbf{a}$ and $\mathbf{b}$ 


\section{Appendix Ground state master equation for optical pumping}

The density matrix $\sigma$ of the ground $6 S_{1 / 2}$ and excited $6 P_{1 / 2}$ states has the form

$\sigma=\left(\begin{array}{c}\sigma_{\mathrm{gg}} \sigma_{\mathrm{ge}} \\ \sigma_{\mathrm{eg}} \sigma_{\mathrm{ee}}\end{array}\right)$

where $\sigma_{a b}=P_{a} \sigma P_{b}$ with $a, b=e, g . \sigma_{g g}$ is identical with $\varrho$ of the main text. It is assumed that the substates inside a level to be degenerate in the sense that their hyperfine and Zeeman structure cannot be resolved by optical means. In the rotating wave approximation the atom light interaction Hamiltonian is then given by

$H_{A L}=\hbar\left(\begin{array}{cc}0 & D_{\mathrm{ge}} \\ D_{\mathrm{eg}} & -\Delta\end{array}\right)$,

where $\Delta=\omega-\omega_{\mathrm{eg}}$ is the detuning of the laser frequency from the optical transition frequency $\omega_{\mathrm{eg}}$. The relaxation processes are described by the rates $\left(\dot{\sigma}_{a b}\right)_{\text {rel }}$ with

$\left(\dot{\sigma}_{e e}\right)_{\text {rel }}=-\Gamma \sigma_{e e}$

$\left(\dot{\sigma}_{\mathrm{eg}}\right)_{\mathrm{rel}}=-1 / 2(\Gamma+\gamma) \sigma_{\mathrm{eg}}$

$\left(\dot{\sigma}_{\mathrm{ge}}\right)_{\mathrm{rel}}=-1 / 2(\Gamma+\gamma) \sigma_{\mathrm{ge}}$

$\left(\dot{\sigma}_{g g}\right)_{\text {rel }}=\Gamma \frac{2 L+1}{L} \sum_{q=-1}^{1}\left(C_{-q}^{1}\right)_{\mathrm{ge}} \sigma_{e e}\left(C_{q}^{1}\right)_{\mathrm{eg}}$.

$1 / \Gamma$ is the lifetime of the $6 P_{1 / 2}$ level and $\gamma$ is the homogeneous width of the optical transition. $L=1$ is the orbital angular momentum of the exited state. Since in this experiment $\gamma \gg \Gamma$, it is possible to neglect the $\Gamma / 2$ terms in the relaxation of the optical coherences.

The Liouville equation for the atom-light interaction is

$\dot{\sigma}=-i / \hbar\left[H_{A L}, \sigma\right]+(\dot{\sigma})_{\text {rel }}$.

Inserting (A.1) and (A.2) into (A.3) and setting $\dot{\sigma}_{\mathrm{eg}}=0$ and $\dot{\sigma}_{\mathrm{ge}}=0$ (adiabatic elimination) yields for $\sigma_{g g}$ and $\sigma_{e e}$ :

$$
\begin{aligned}
\dot{\sigma}_{e e}= & D_{\mathrm{eg}} \sigma_{g g} D_{\mathrm{ge}} \frac{2 \gamma}{\Delta^{2}+(\gamma / 2)^{2}}-\Gamma \sigma_{e e} \\
\dot{\sigma}_{g g}= & -i\left(\frac{D_{\mathrm{ge}} D_{\mathrm{eg}} \sigma_{g g}}{\Delta+i \gamma / 2}-\frac{\sigma_{g g} D_{\mathrm{ge}} D_{\mathrm{eg}}}{\Delta-i \gamma / 2}\right) \\
& +\left(\dot{\sigma}_{g g}\right)_{\mathrm{rel}} .
\end{aligned}
$$

Here all terms describing stimulated transitions from the excited state to the ground state have been neglected, which is justified because of the very large homogeneous linewidth $(10 \mathrm{~nm})$ and the modest laser intensities (several $10 \mathrm{~mW} / \mathrm{cm}^{2}$ ) used. Since the lifetime of the excited state $\left(1 / \Gamma \approx 10^{-8} \mathrm{~s}\right)$ is very short, the evolution of $\sigma_{e e}$ under the influence of the magnetic fields can also be neglected. In the steady state $\dot{\sigma}=0$ it is possible to solve (A.4) for $\sigma_{e e}$ and insert the result into $\left(\dot{\sigma}_{g g}\right)_{\text {rel }}$ of (A.5). This gives

$$
\begin{aligned}
\sigma_{e e}= & \frac{1}{\Gamma} \frac{\gamma}{\Delta^{2}+(\gamma / 2)^{2}} D_{\mathrm{eg}} \sigma_{g g} D_{\mathrm{ge}} \\
\dot{\sigma}_{g g}= & -i\left(\frac{D_{\mathrm{ge}} D_{\mathrm{eg}} \sigma_{g g}}{\Delta+i \gamma / 2}-\frac{\sigma_{g g} D_{\mathrm{ge}} D_{\mathrm{eg}}}{\Delta-i \gamma / 2}\right) \\
& +\frac{3 \gamma}{\Delta^{2}+(\gamma / 2)^{2}} \sum_{q=-1}^{1}\left(C_{-q}^{1}\right)_{\mathrm{ge}} D_{\mathrm{eg}} \sigma_{g g} D_{\mathrm{ge}}\left(C_{q}^{1}\right)_{\mathrm{eg}} .
\end{aligned}
$$

The fluorescence rate $\mathcal{F}$ is then calculated to be

$\mathcal{F}=\Gamma \operatorname{tr}\left(\sigma_{e e}\right)=\frac{\gamma}{\Delta^{2}+(\gamma / 2)^{2}} \operatorname{tr}\left(D_{\mathrm{eg}} \sigma_{g g} D_{\mathrm{ge}}\right)$.

Setting $\Delta=0$ in (A.6) and (A.7) yields the expressions (10) and (11) of the main text.

ACKNOWLEDGEMENTS This work was supported by a grant from the Schweizerischer Nationalfonds. We thank S. Ulzega for helping during the experimental preparation and data recording.

\section{REFERENCES}

1 D. Nettels, R. Müller-Siebert, S. Ulzega, A. Weis: accepted for publication by Appl. Phys. B

2 A.S. Pazgalev, E.B. Alexandrov: Optics Spectrosc. 86, 6 (1999) S.I. Kanorsky, M. Arndt, R. Dziewior, A. Weis, T.W. Hänsch: Phys. Rev. B 49, 3645 (1994)

3 S.I. Kanorsky, M. Arndt, R. Dziewior, A. Weis, T.W. Hänsch: Phys. Rev. B 49, 3645 (1994)

4 S. Lang, S.I. Kanorsky, T. Eichler, R. Müller-Siebert, T.W. Hänsch, A. Weis: Phys. Rev. A 60, 3867 (1999)

5 M. Arndt, S.I. Kanorsky, A. Weis, T.W. Hänsch: Phys. Rev. Lett. 74, 1359 (1995)

6 S.I. Kanorsky, S. Lang, S. Lücke, S.B. Ross, T.W. Hänsch, A. Weis: Phys. Rev. A 54, R1010 (1996)

7 H. Salwen: Phys. Rev. 99, 1274 (1955)

8 J. Hermann, S. Swain: J. Phys. B 10, 1815 (1977)

9 W. Happer: Rev. Mod. Phys. 44, 169 (1972)

10 W. Happer: Phys. Rev. B 1, 2203 (1970)

11 S. Lang, S.I. Kanorsky, M. Arndt, S.B. Ross, T.W. Hänsch, A. Weis: Europhys. Lett. 30, 233 (1995)

12 A. Abragam: The principles of nuclear magnetism (Clarendon Press, Oxford 1961)

13 V.J. Minkiewicz, T.A. Kitchens, F.P. Lipschultz, R. Nathans, G. Shirane: Phys. Rev. 174, 267 (1968)

14 S. Lang: Ph.D. thesis, Ludwig-Maximilians-Universität München (1997)

15 K. Blum: Density matrix theory and application (Plenum Press, NY 1996) 2nd ed.

16 M. Weissbluth: Photon-Atom Interactions (Academic Press, London 1989)

17 Mathematica (Wolfram Research, Inc., Il USA 2002) version 4.2.1.0

18 R. Müller-Siebert, D. Nettels, A. Weis: to be submitted 